\title{
Intestinal permeability changes and excretion of micro-organisms in stools of infants with diarrhoea and vomiting
}

\author{
L T WEAVER, P D CHAPMAN, C R MADELEY, M F LAKER, AND R NELSON
}

Departments of Child Health, Virology, and Clinical Biochemistry; Royal Victoria Infirmary, Newcastle upon Tyne

SUMmaRY. The relation between diarrhoea and vomiting, the excretion of stool microorganisms, and the passive intestinal permeability in 20 infants living in a deprived urban area was studied prospectively from birth to age 6 months. Intestinal permeability was measured from the ratio of lactulose to mannitol recovered in the urine of infants receiving feeds containing both markers. Micro-organism excretion was found to occur in both the presence and absence of gastrointestinal symptoms, but a significantly higher mean intestinal permeability was recorded in those infants with symptoms and organisms in the stool than in those with neither. An increased intestinal permeability may be a sign of mucosal damage by intestinal micro-organisms.

The relation between the excretion of viruses in the stool and disease of the gastrointestinal tract of infants is not clear. Scott et $a l,{ }^{1}$ in a prospective community study of infants from a deprived area of Glasgow, found that virus excretion was frequently unaccompanied by illness, and were unable to describe a typical syndrome in association with any of the viruses observed. Asymptomatic virus excretion was reported in children in hospital by Walther et al ${ }^{2}$ who found rotavirus in the stools of $14.5 \%$ of infants of less than 6 months, with neither diarrhoea nor vomiting.

Although Bishop et $a^{3}$ found virus particles in the duodenal epthelial cells of six of nine children with acute non-bacterial gastroenteritis, resolving with recovery, and Barnes and Townley ${ }^{4}$ reported mucosal changes, including blunting and shortening of the villi, in association with depressed disaccharidase activity, the latter workers were unable to relate the degree of damage to the clinical severity of the illness. Harrison and Walker-Smith ${ }^{5}$ found no correlation between clinical lactose tolerance, lactase concentrations, and small intestinal mucosal morphology in children with postgastroenteritis milk intolerance.

Xylose uptake may be depressed in gastroenteritis, ${ }^{6}$ though normal in the presence of abnormal mucosal architecture. ${ }^{7}$ Mavromichalis $e t a l^{8}$ showed depressed xylose uptake in nine infants with gastroenteritis who harboured rotavirus or advenovirus in their jejunal aspirates, but not in those who excreted virus in their stools alone.

To investigate this puzzling relation between the clinical features of gastroenteritis, the excretion of possible intestinal pathogens, and changes in mucosal structure and function we performed a prospective community study of infants from a deprived area of Tyneside.

\section{Subjects and methods}

Infants were selected after birth at a single maternity hospital in Tyneside according to the following criteria designed to identify those at risk of gastrointestinal infection:

(1) Infants whose mothers planned to feed them with an artificial milk formula.

(2) Residence in one area of Tyneside with high unemployment and population density, low socioeconomic status, and poorly maintained housing with inadequate environmental and social services.

(3) One or more young children in the household.

Informed consent was obtained from the parents of all infants in the study, which was performed with the approval of the local ethical committee. After discharge from the maternity unit a research health 
visitor (PDC) visited each infant at least once weekly to record domestic and social conditions, feeding patterns, and gastrointestinal symptoms; to collect stools for microbiological analysis; and perform tests of intestinal permeability.

Domestic and social conditions. A record was made of the condition of the household and mother's standard of hygiene, especially in feed preparation, using a score of 1 (worst) to 5 (best), the number of other children and animals in the household, the temperature and dampness (using a protimeter) of the infant's sleeping accommodation, and the pattern and composition of infant feeding.

Gastrointestinal symptoms. The frequency and duration of episodes of diarrhoea or vomiting, or both were recorded. Diarrhoea was defined as a change in bowel habit with either an increase over the usual frequency of stools or at least one watery stool, and vomiting as the regurgitation of an appreciable volume of feed outside the normal feeding pattern. A sequential record of infants' weights was kept.

Microbiological analysis of stools. Stool samples were obtained weekly for microbiological analysis, and more frequently during episodes of diarrhoea and vomiting or organism excretion; stored at $-20^{\circ} \mathrm{C}$; and transported to the laboratory within 36 hours of collection. All stools were examined virologically by electron microscopy and culture, ${ }^{9}$ and bacteriologically by routine methods, including culture for campylobacters and yersinias, if the infant had diarrhoea or vomiting. Stools were not examined for parasites.

Intestinal permeability tests. These were performed every six weeks or more frequently if the infant had symptoms or a potential intestinal pathogen was identified. A dual marker, steady state method was used. ${ }^{10}$ Lactulose and mannitol were chosen as markers because both are present in some infant feeds, both resist hydrolysis by intestinal enzymes, and both are passively absorbed by different routes across the intestinal wall-lactulose by paiacellular pathways and mannitol transcellularly. Neither marker is metabolised after uptake, and both are excreted wholly and solely in the urine where recovery approaches $100 \% .{ }^{11} 12$ Urinary excretion is therefore a measure of intestinal absorption.

When the two markers are combined in test feeds, the effects of variables such as rate of gastric emptying, intestinal transit, distribution within the body, and renal clearance on the results apply equally to both. Infants received regular three to six hourly feeds containing the markers in a fixed concentration ratio of $5: 1$. Test feeds were prepared by mothers with the help of the research health visitor (PDC) by adding $5 \mathrm{ml}$ of an aqueous solution containing lactulose $(4 \mathrm{~g} / 100 \mathrm{ml})$ and mannitol $(0 \cdot 8$ $\mathrm{g} / 100 \mathrm{ml}$ ) to every $100 \mathrm{ml}$ of made up feed. The granulated milks used contain negligible amounts of the markers ${ }^{10}{ }^{13}$ and the addition of $200 \mathrm{mg}$ lactulose and $40 \mathrm{mg}$ mannitol per $100 \mathrm{ml}$ feed is within the range of concentrations of the markers found in many 'ready to feed' liquid formulae. No adverse effects were seen with the use of these test feeds.

After 24 hours of regular test feeds, when a steady state of marker input and output had been achieved, a random urine sample of at least $2 \mathrm{ml}$ was obtained. Solids were excluded from the diet during this period. Urine samples were stored at $-20^{\circ} \mathrm{C}$ with $0 \cdot 1 \mathrm{ml} 10 \%$ sodium merthiolate, and marker analysis was performed by gas liquid chromatography. ${ }^{12} 14$

Intestinal permeability was expressed as a urinary lactulose:mannitol excretion ratio calculated from amounts of the markers present in urine samples compared with the fixed ratio in test feeds. A normal range of less than 0.55 was taken from a study of 24 infants on different feeds (breast milk, cows' milk, and soya formulae) aged six weeks.

Gastrointestinal symptoms, stool micro-organisms, and tests of intestinal permeability were recorded or performed between Mondays and Fridays each week, but not always on the same day. In the analysis of the results correlation between these three variables was made whatever their chronological relations within a single week. The Student's $t$ test was used to determine the significance of the results.

Conversion factors: lactulose $1 \mathrm{mg} / \mathrm{l}=2.92 \mathrm{mmol} / \mathrm{l}$; mannitol $1 \mathrm{mg} / \mathrm{l}=5.49 \mathrm{mmol} / \mathrm{l}$.

\section{Results}

Social and domestic data (Table 1). 20 infants were studied from birth to 6 months of age. Their mean birthweight was $3.2 \mathrm{~kg}$ (range $2 \cdot 2$ to $4.2 \mathrm{~kg}$ ). Fourteen were boys and six girls. Nine were illegitimate, but in only two cases were the fathers not at home. Sixteen of these 18 fathers were unemployed. The mean score for domestic conditions was 2.9 (range 1 to 4 ), and for hygiene in feed preparation 2.8 (range 1 to 5). There was a significant inverse relation between frequency of episodes of diarrhoea and vomiting and scores for domestic conditions $(\mathrm{P}<0.01)$ and hygiene in feed preparation $(\mathrm{P}<0 \cdot 02)$.

The mean number of other children in the households was 2 (range 1 to 5), and animals 1.2 (range 0 to 5). The latter included $18 \mathrm{dogs}$, one cat, two rabbits, two horses, one goat, two budgerigars, 
and two lofts of pigeons. There was no significant relation between these variables and the incidence of diarrhoea and vomiting.

The temperature of the sleeping accommodation of all infants was above $10^{\circ} \mathrm{C}$, and dampness of walls less than $10 \%$ moisture in all but one case. The weights of two infants at birth were below the 3rd centile but rose to within the normal range within a month of delivery, and no infant fell below this centile during the course of the study. The weight of one infant rose above the 97 th centile. One infant was admitted to hospital during the course of the study with campylobacter gastroenteritis and two with upper respiratory tract infections. Mean age of introduction of first solid feeds was 10.5 weeks (range 6 to 25), but there was no significant relation between this and the incidence of diarrhoea and vomiting or pattern of intestinal permeability changes. A fuller description and analysis of the social and domestic data will be published elsewhere. ${ }^{15}$

Gastrointestinal symptoms (Table 1). Forty four episodes of vomiting or diarrhoea, or both, were recorded (mean 2.2 per infant, range 0 to 7 ), and during 21 of these, excretion of micro-organisms was also present $(48 \%)$, which was one week per infant. The organisms associated with gastrointestinal symptoms are shown in Table 2 . On 22 occasions tests of intestinal permeability were performed in

Table 1 Summary of results

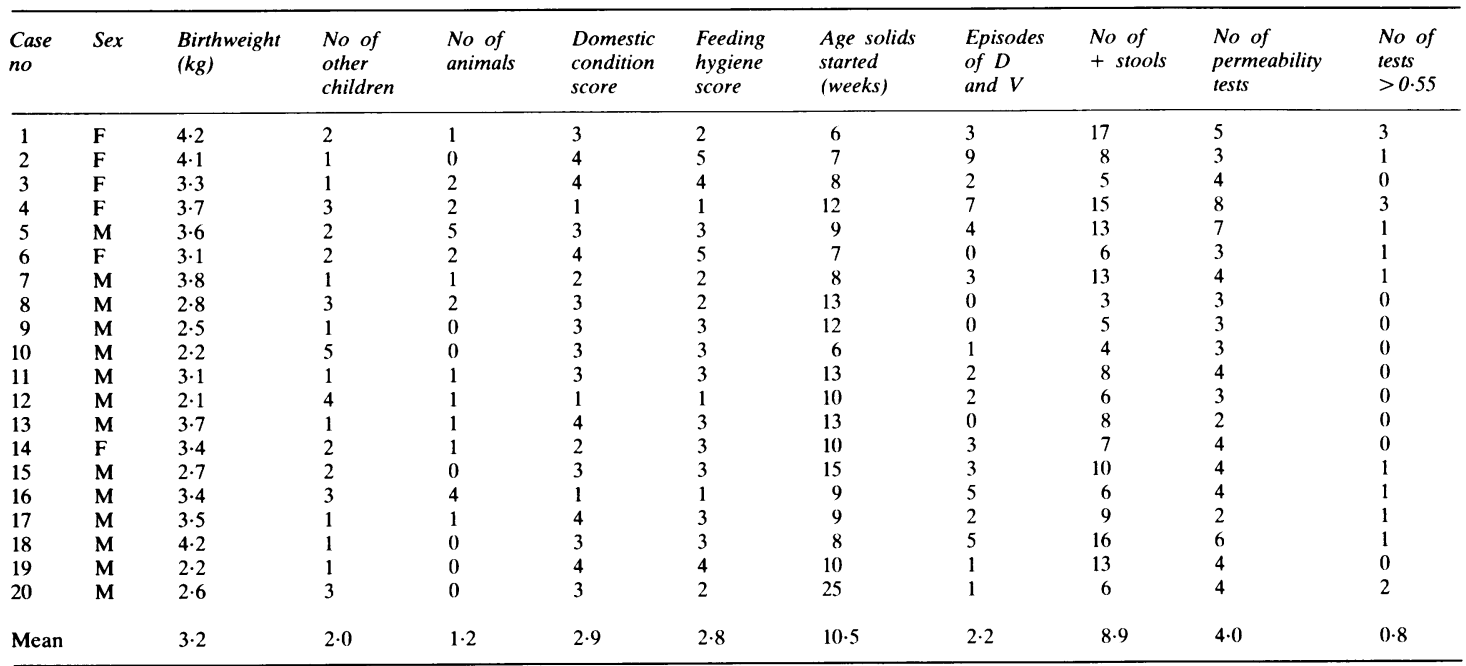

$\mathrm{D}$ and $\mathrm{V}=$ diarrhoea and vomiting.

Table 2 Micro-organisms identified in the stool, with number of each type in brackets, in the presence and absence of gastrointestinal symptoms

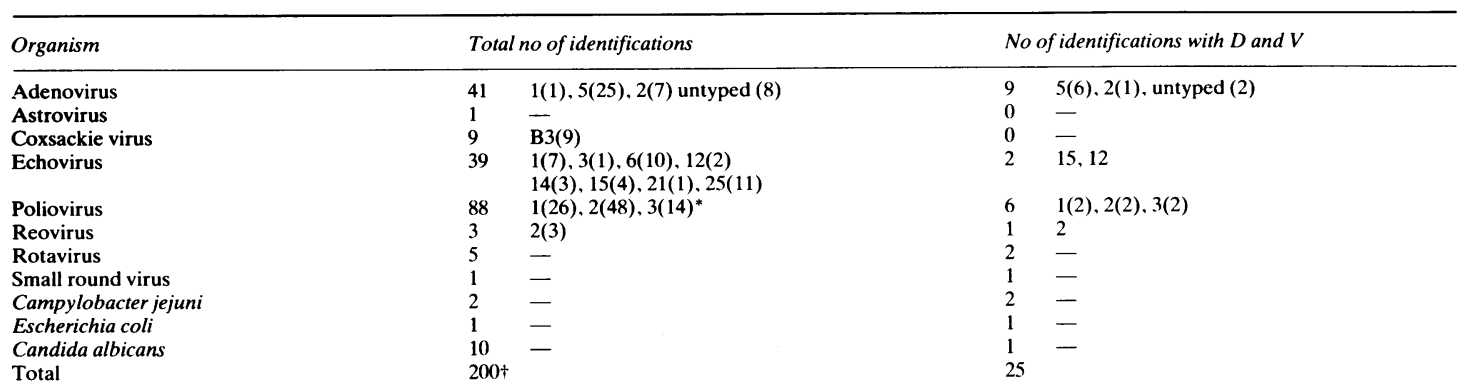

*On 14 occasions more than one strain present in stool sample.

+On 8 occasions more than one organism present in stool sample.

Untyped: observed by electron microscopy but failed to grow on culture.

$\mathrm{D}$ and $\mathrm{V}=$ diarrhoea and vomiting. 
the same week as vomiting or diarrhoea, or both, occurred (Fig. 1).

Stool micro-organism excretion (Table 2). Samples were obtained for microbiological analysis on 426 weeks from a theoretical total of $520(82 \%)$. The discrepancy was due to maternal collection and transport failures, late recruitment to the study, and holidays. Potentially pathogenic micro-organisms were identified on 178 weeks $(42 \%)$, which was 8.9 weeks per infant. On $88 \%$ of the weeks that organisms were found in the stools no gastrointestinal symptoms were present. On eight occasions more than one organism was found, and on 14 occasions more than one type of poliovirus, making a total number of identifications of 200 . Thirty six stool samples proved unsuitable for virological culture because of overgrowth of bacteria or fungi. In none of these was a virus identified by electron microscopy.

Adenovirus type 5 was the commonest organism found in association with diarrhoea and vomiting, and on only two of the five occasions that rotavirus was found were symptoms present. Prolonged excretion for more than three weeks of adenovirus (three infants), echovirus (three infants) and poliovirus (eight infants) occurred. Poliovirus was the micro-organism found most frequently in asymptomatic infants. The 18 infants who received polio vaccine during the study all excreted the virus, plus two infants who did so before immunisation. The

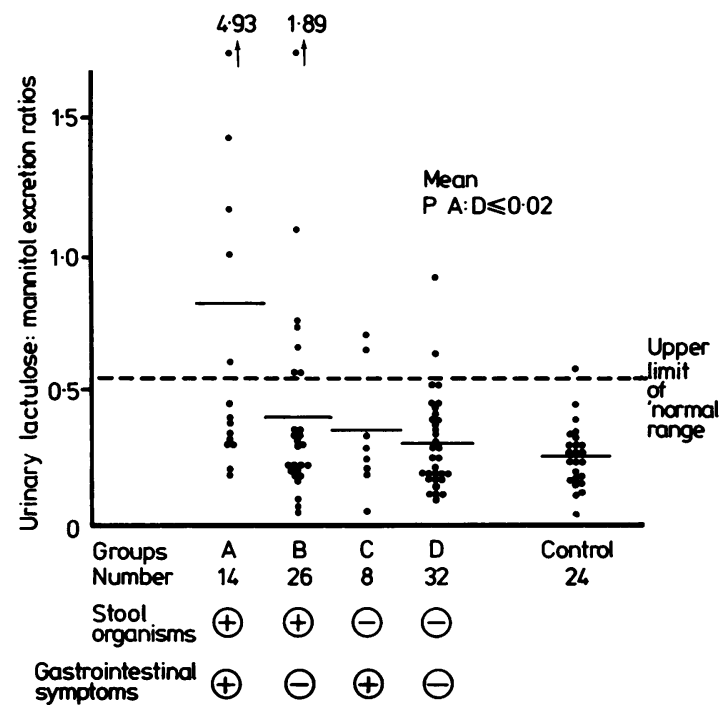

Fig. 1 Urinary marker excretion ratios in the presence and absence of stool micro-organism excretion and gastrointestinal symptoms in all infants.

latter were both in close contact with recently immunised children.

Tests of intestinal permeability (Figs. 1 and 2). Eighty tests of intestinal permeability were per-

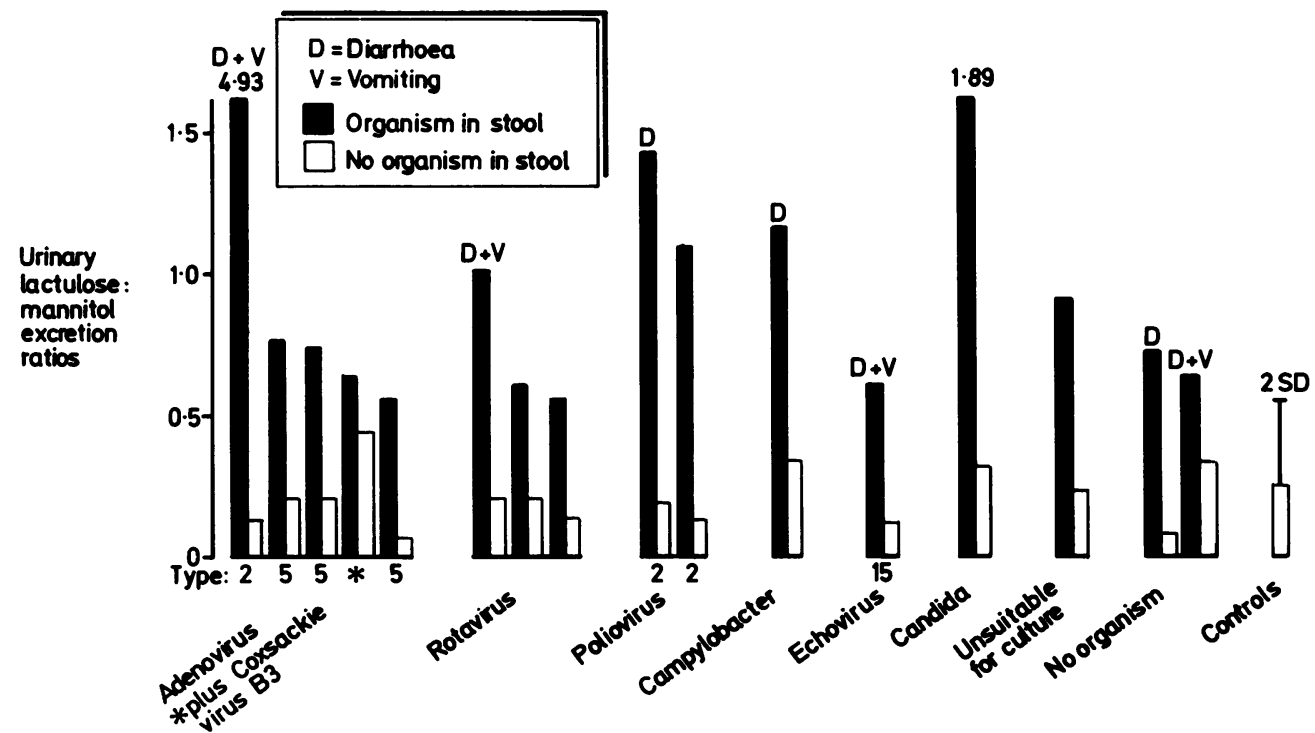

Fig. 2 Urinary marker excretion ratios in the presence and absence of stool micro-organism excretion and gastrointestinal symptoms in infants with intestinal permeability ratios above normal range. 
formed (mean 4 per infant). Infants were divided into four groups for analysis:

(1) Group A consisted of 14 infants with diarrhoea or vomiting, or both, and with potentially pathogenic micro-organisms identified in the stool.

(2) Group B comprised 26 infants with neither diarrhoea nor vomiting but with potentially pathogenic micro-organisms identified in the stool.

(3) Group C comprised eight infants with diarrhoea or vomiting, or both, but no potentially pathogenic micro-organisms identified in the stool.

(4) Group D comprised 32 infants with neither diarrhoea nor vomiting nor potentially pathogenic micro-organisms identified in the stool.

There was a significant difference $(P<0.02)$ between the mean permeability ratios of groups $A$ and $D$. An intestinal permeability ratio of less than 0.55 was recorded in nine of group A, 19 of group B, six of group C, and 30 of group D (Fig. 1).

The results obtained from those 16 infants whose permeability ratios were above the normal range are shown in Fig. 2. The first upright bar for each infant represents the urinary lactulose:mannitol excretion ratio recorded during excretion of a micro-organism with and without diarrhoea or vomiting, or both, and the second bar that after cessation of organism excretion (if present) or diarrhoea or vomiting, or both. On one occasion possible virus identification was prevented by bacterial overgrowth, and on two occasions no organisms were identified in the presence of diarrhoea and vomiting. On one of the five occasions that adenovirus (untyped) was identified Coxsackie virus type B3 was also found. An increased intestinal permeability ratio was recorded the following week.

There was no significant relation between the prescription of antimicrobials or other medication, and either gastrointestinal symptoms or alterations in intestinal permeability.

\section{Discussion}

In spite of a high incidence of adverse environmental factors (Table 1) and a wide range of microorganisms excreted (Table 2) only one infant required admission to hospital with gastroenteritis, and no baby failed to thrive during the course of the study. This may, in part, have been due to the regular attention and support of the research health visitor (PDC). There was, however, a significant relation between adverse environmental circumstances and the frequency of episodes of diarrhoea and vomiting.

While stool micro-organism excretion was recorded on 8.9 weeks per infant, and episodes of diarrhoea or vomiting, or both, on 2.2 weeks per infant, the identification of stool micro-organisms in association with symptoms occurred on only one week per infant. These figures are similar to those of Scott $e^{a} a^{1}$ who reported that only $41 \%$ of stool virus identifications were associated with episodes of illness, in a cohort of 27 infants from a comparable area of inner city Glasgow. They also observed that adenovirus was the virus most commonly detected, and that repeated excretion was not unusual.

The absence of micro-organism excretion in the stool of $52 \%$ of infants we studied with diarrhoea or vomiting, or both, may have been due to the limitations of the analytic methods. Electron microscopy, at present the only method capable of detecting every kind of virus in the stool, is insensitive when virus is present in less than a million particles per gram of faeces. ${ }^{9}$ Not all viruses observed by electron microscopy, however, grow in cell culture, and bacterial and fungal overgrowth may interfere. Consequently failure to detect virus in the stool does not necessarily indicate absence of virus, even though some episodes of diarrhoea and vomiting may have been due to non-infective causes.

Conversely the identification of virus in the stool of an infant with diarrhoea and vomiting does not necessarily constitute causation of those symptoms. Scott $e t a^{1}$ observed a rapidly changing flora in sequential examinations of stools from the same infant, and as we have found, more than one potentially pathogenic organism may be identified in the stool of infants with symptoms. The absence of symptoms in the presence of potentially pathogenic micro-organisms in the stool further supports the contention that virus excretion is not necessarily indicative of gastrointestinal disease.

We have shown that stool micro-organism excretion may occur with and without gastrointestinal symptoms and with and without alterations in intestinal permeability. An enhanced intestinal permeability ratio occurred with micro-organism excretion or diarrhoea and vomiting, or both, in $94 \%$ of cases. In the single case in which an enhanced permeability ratio was recorded in the absence of either micro-organism excretion or symptoms, stool viral culture was contaminated by bacterial overgrowth. We suggest that an enhanced urinary lactulose:mannitol excretion ratio is a sign of mucosal damage.

The use of a combination of two markers of different sizes to measure changes in small intestinal permeability has proved a sensitive and specific method in both paediatric and adult studies. It is thought that the passage of inert disaccharides such as lactulose (molecular radius $0.54 \mathrm{~nm}$ ) and cellobiose (molecular radius $0.50 \mathrm{~nm}$ ) across the 
mucosa is restricted to paracellular pathways-the intercellular spaces and extrusion zones and other sites of enterocyte loss, while smaller markers such as mannitol and L-rhamnose (molecular radius $0 \cdot 40$ $\mathrm{nm}$ ) pass predominantly transcellularly via aqueous pores in the cell membrane. Such a hypothesis supposes a low incidence of the larger pathways and a high incidence of the smaller. In villous atrophy a decline in surface area leads to a corresponding reduction in the aqueous pore population by which only the smaller markers may pass, and in mucosal damage to an increase in paracellular pathways available for uptake of larger markers. ${ }^{16}$

In untreated coeliac disease in childhood there is a significant rise in urinary lactulose:mannitol excretion ratios, due mainly to depressed mannitol uptake, while in childhood Crohn's disease it is due to enhanced lactulose absorption. ${ }^{17}$ In children with acute lymphoblastic leukaemia receiving cytotoxic therapy, both mechanisms are affected. ${ }^{18}$ Weaver $e t$ $a l^{19}$ showed a sudden increase in lactulose absorption in three preterm infants with incipient necrotising enterocolitis, returning to normal with recovery. Using lactulose and L-rhamnose Menzies ${ }^{16}$ reported an increased urinary lactulose:L-rhamnose ratio in 14 infants with viral gastroenteritis (13 with rotavirus and one adenovirus) returning to normal with recovery.

Although bacteria as well as viruses may be present in the small intestine of infants with acute gastroenteritis, ${ }^{20}$ the saturating doses of markers passing down the gut (over $95 \%$ of ingested marker is unabsorbed) and the equal susceptibility of lactulose, mannitol, and L-rhamnose to hydrolysis by intestinal micro-organisms, ${ }^{10}$ make it unlikely that a selective bacterial degradation of mannitol could explain the changes we have observed in urinary lactulose:mannitol excretion ratios.

In acute viral gastroenteritis, where disruption of mucosal integrity may accompany villous $\operatorname{loss}^{4}$ expression of the intestinal permeability as a ratio of lactulose:mannitol absorbed is particularly appropriate and sensitive. Excretion of micro-organisms in the stool is not proof of colonisation or damage of the small intestine by such organisms, even in the presence of diarrhoea and vomiting. These preliminary data suggest, however, that in those infants whose permeability ratios were raised, the small intestinal mucosa may have been damaged by microorganisms. Further studies combining the examination of jejunal aspirates and mucosal biopsies for micro-organisms, with measurement of passive intestinal permeability are needed, both in children with acute gastroenteritis and apparently healthy excretors of potential intestinal pathogens. The use of the dual marker, steady state method with continuous test feeding would allow sequential changes in mucosal permeability to be correlated more tightly with episodes of diarrhoea, vomiting, and micro-organism excretion, and closer definition of the structural and functional changes in the intestinal mucosa of infants with gastroenteritis.

We thank the staff of the Departments of Virology, Royal Victoria Infirmary, and Microbiology, Dunston Hill Hospital for their help, the Regional and Area Health Authorities for supporting PDC and LTW, and the parents of all the infants studied.

\section{References}

${ }^{1}$ Scott TM, Madeley CR, Cosgrove BP, Stanfield JP. Stool viruses in babies in Glasgow 3. Community studies. J Hyg Camb 1979;83:469-85.

2 Walther FJ, Bruggeman C, Daniels-Bosman MSM, et al. Symptomatic and asymptomatic rotavirus infections in hospitalized children. Acta Paediatr Scand 1983;72:659-63.

3 Bishop RF, Davidson GP, Holmes IH, Ruck BJ. Virus particles in epithelial cells of duodenal mucosa from children with acute non-bacterial gastroenteritis. Lancet 1973;ii:1281-3.

4 Barnes GL. Townley RRW. Duodenal mucosal damage in 31 infants with gastroenteritis. Arch Dis Child 1973;48:343-9.

5 Harrison M, Walker-Smith JA. Reinvestigation of lactose intolerant children: lack of correlation between continuing lactose intolerance and small intestinal morphology, disaccharidase activity, and lactose tolerance tests. Gut 1977;18:48-52.

6 Buts J-P, Morin CL, Roy CC, Weber A, Bonin A. One-hour blood xylose test: a reliable index of small bowel function. J Pediatr 1978;90:729-33.

${ }^{7}$ Christie DL. Use of the one hour blood xylose test as an indicator of small bowel mucosal disease. J Pediatr 1978;92: $725-8$.

${ }^{8}$ Mavromichalis J, Evans N, McNeish AS, Bryden AS, Davies HA, Flewett TH. Intestinal damage in rotavirus and adenovirus gastroenteritis assessed by D-xylose malabsorption. Arch Dis Child 1977;52:589-91.

9 Madeley CR, Cosgrove BP, Bell EJ, Fallon RJ. Stool viruses in babies in Glasgow 1. Hospital admissions with diarrhoea. J Hyg Camb 1977;78:261-73.

10 Weaver LT, Laker MF, Nelson R. Intestinal permeability in the newborn. Arch Dis Child 1984;59:236-41.

1 Laker MF, Menzies IS. Increase in human intestinal permeability following ingestion of hypertonic solutions. J Physiol 1977;265:881-94.

12 Laker MF, Bull HJ, Menzies IS. Evaluation of mannitol for use as a probe marker of gastrointestinal permeability in man. Eur $J$ Clin Invest 1982;12:485-91.

13 Beach RC, Menzies IS. Lactulose and other non-absorbable sugars in infant milk feeds. Lancet 1983;i:425-6.

14 Laker MF. Estimation of disaccharides in plasma and urine by gas-liquid chromatography. J Chromatogr 1979;163:9-18.

15 Chapman PD, Weaver LT. Gastroenteritis in infancy: a community study. Health Visitor 1985; in press.

16 Menzies IS. Transmucosal passage of inert molecules in health and disease. In: Skadhauge E, Heintze K, eds. Intestinal absorption and secretion, Falk symposium 36. Boston: MTP Press, 1984;527-43.

17 Pearson ADJ, Eastham EJ, Laker MF, Craft AW, Nelson R. Intestinal permeability in children with Crohn's disease and coeliac disease. Br Med J 1982;285:20-1.

18 Pearson ADJ, Craft AW, Pledger JV, Eastham EJ, Laker MF, 
Pearson CL. Small bowel function in acute lymphoblastic leukaemia. Arch Dis Child 1984;59:460-5.

19 Weaver LT, Laker MF, Nelson R. Enhanced intestinal permeability in preterm babies with bloody stools. Arch Dis Child 1984;59:280-1.

20 Challacombe DN, Richardson JM, Rowe B, Anderson CM. Bacterial microflora of the upper gastrointestinal tract in infants with protracted diarrhoea. Arch Dis Child 1974;49: 270-7.

Correspondence to Dr L T Weaver, Dunn Nutritional Laboratory, Downham's Lane, Milton Road, Cambridge CB4 1XJ.

Received 3 January 1984

\section{Fifty years ago}

\section{Papular urticaria (lichen urticatus)}

B C TATE (Birmingham) - Arch Dis Child 1935;10:27-48

'1. Papular urticaria is a definite and distinct syndrome, separable from urticaria and from Hebra's prurigo.

2. It is a manifestation of allergy.

3. It is essentially a disorder of the first four years of life.

4. It is aggravated by heat, and by a diet containing too much carbohydrate.

5. Digestive disorders, teething and mild febrile disturbances possibly predispose to papular urticaria, but such conditions are of very minor importance in its aetiology. Rickets plays no part.

6. There is no satisfactory evidence of any underlying diathesis.

7. The exciting agent is not a food.

8. The exciting agent is something connected with the patients' home environment but its precise nature remains obscure. It is not bedding or clothes, but it can be carried on these articles. It is not vermin, animal-size, nor an antigen derived from human beings or domestic animals. Sensitivity to house dust has not been definitely excluded.'

(Dr Tate rejected the idea of flea or insect bites as a possible cause of this itchy nuisance in his detailed and careful investigation mostly it seems because the condition occurred 'in all grades of society'. The fleas of the nation's domestic animals and birds, however, have a notorious disregard for social stratification. Dr Tate later became Honorary Physician in charge of the Skin Department at the Children's Hospital, Birmingham among other appointments, and also served as President of the British Association of Dermatologists. PAMEla A Davies.) 